\title{
Focused-Ion-Beam Fabrication of Slanted Grating Couplers in Silicon-on-Insulator Waveguides
}

\author{
Jonathan Schrauwen, Student Member, IEEE, Frederik Van Laere, Student Member, IEEE, \\ Dries Van Thourhout, Member, IEEE, and Roel Baets, Fellow, IEEE
}

\begin{abstract}
We have designed and fabricated an efficient grating coupler for coupling light between optical fibers and silicon-on-insulator waveguides. The coupler consists of 88-nm-wide slits, etched at an angle of $58^{\circ}$ to the surface normal. They are defined by direct etching with a focused ion beam, using iodine gas and an alumina hard mask. The measured efficiency is $46 \%$.
\end{abstract}

Index Terms-Fiber coupler, focused-ion-beam (FIB), slanted grating coupler.

\section{INTRODUCTION}

$\mathbf{T}$ HE silicon-on-insulator (SOI) platform is a promising candidate for future ultracompact photonic integrated circuits because of its compatibility with complementary metal-oxide-semiconductor technology [1]. The high index contrast in this material system allows for the fabrication of short waveguide bends and, therefore, circuits with a high degree of integration. Coupling between a high index contrast waveguide and an optical fiber is primordial, but difficult due to the large mismatch between the optical modes. In literature, one proposes out-of-plane grating couplers as a solution to this problem [2]. Due to the large index contrast, the gratings are very compact and broadband. Furthermore, the out-of-plane approach makes polished facets unnecessary and enables wafer-scale testing of integrated optical circuits.

Most grating couplers up to now were fabricated with standard techniques based on optical lithography [3], [4]. Another approach is the direct etching of grating couplers in predefined silicon waveguides. This enables in situ analysis of integrated circuits. A promising method for the direct etching approach is focused-ion-beam (FIB), a common tool for device analysis and modification in electronics.

Microfabrication with FIB consists of hitting a substrate locally with high energy ions; in most commercial systems, like the FEI Dualbeam 600 used in this work, these are gallium ions with energies around $30 \mathrm{keV}$. In crystalline substrates, this process induces lattice damage, makes the top layer amorphous, and implants ions deeper into the substrate [5], [6]. Therefore,

Manuscript received January 26, 2007. This work was supported in part by the European Union through the Network of Excellence ePIXnet, in part by the Belgian IAP-PHOTON Network, and in part by the Fund for Scientific Research (FWO).

The authors are with the Department of Information Technology (INTEC), Ghent University, B-9000 Gent, Belgium (e-mail: Jonathan.Schrauwen@ intec. ugent.be; Frederik.VanLaere@ intec.ugent.be; Dries.VanThourhout@intec. ugent.be; Roel.Baets@intec.ugent.be).

Digital Object Identifier 10.1109/LPT.2007.897293

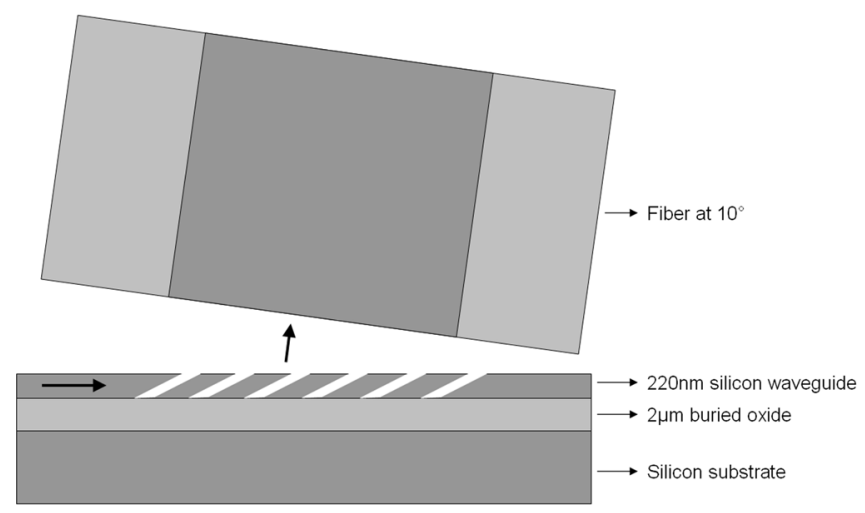

Fig. 1. Schematic drawing of the slanted fiber coupler.

direct etching of grating couplers with FIB has proven unsuccessful [7]. Nevertheless, there are fabrication strategies to minimize optical losses, such as high temperature annealing [8], or the use of a protective mask and a chemical etchant [9]. When carefully adopting these strategies, FIB is a tool that offers rapid prototyping flexibility, enables oblique etching, and has a sub-100-nm resolution. We will explore this unique combination for the fabrication of slanted grating couplers.

It was shown that shallow grating couplers can be etched with FIB [9]. However, these couplers require a two-step etching process and have a moderate coupling efficiency of $24 \%$. An approach to boost the efficiency is the use of slanted slits that are etched entirely through the waveguide layer [10], as depicted in Fig. 1. In this letter, we present the design and fabrication of a slanted grating coupler, combining the flexibility of FIB with the higher efficiency of slanted grating couplers. In Section II, we discuss the design of a slanted fiber coupler, in Section III, we report on the fabrication with FIB, and in Section IV, we present the measurement results.

\section{Simulation}

Regular shallow gratings in SOI are limited in efficiency due to diffraction of the first order into the substrate. This limit can be circumvented by a bottom distributed Bragg reflector mirror [2], or by a bottom gold mirror [3]. However, these greatly complicate the fabrication of integrated circuits in SOI. Another method to enhance the coupling to the upward first order is the use of slanted facets, in analogy to a blazed grating. Because it is difficult to control the depth of the slanted slits with FIB, we have chosen to design a slanted grating with slits through the entire top silicon layer. We use SOI wafers with 220-nm top silicon and $2-\mu \mathrm{m}$ buried oxide (Fig. 1). The gratings were simulated with the finite-difference time-domain (FDTD) method. 


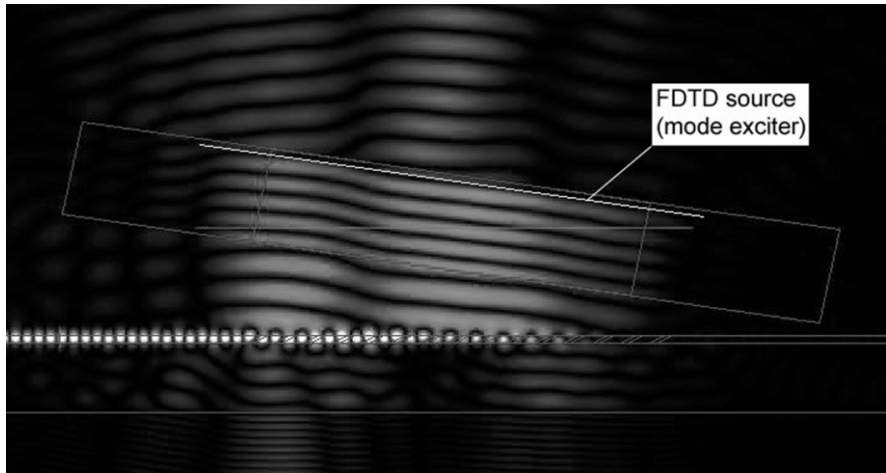

Fig. 2. FDTD simulation of the optimal grating with $64 \%$ efficiency. The plot shows the field pattern at a wavelength of $1550 \mathrm{~nm}$. The mode exciter is located in the fiber, the mode sensor in the SOI waveguide.

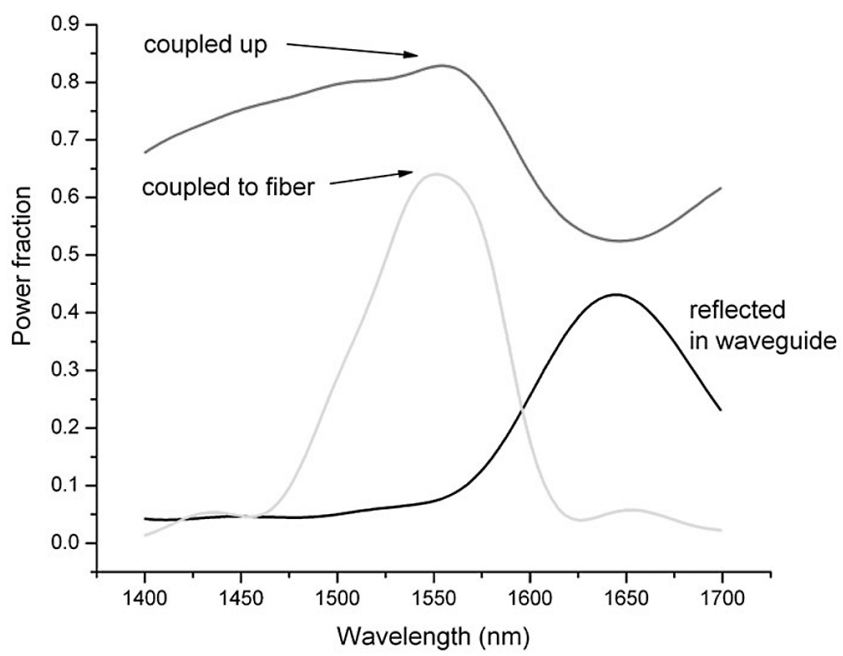

Fig. 3. Result of the slanted coupler optimization with an FDTD simulation. The curves present the power fractions coupled upwards, coupled into the fiber mounted at $10^{\circ}$, and reflected back into the waveguide.

The optimization was done by first scanning the complete parameter space, followed by a local quasi-Newton optimization. Although one can in principle couple light upwards by total internal reflection on a single slanted facet, there will be little overlap with the mode in the fiber in that case. To ensure more overlap with the large fiber mode, one needs to couple light upwards in a distributed way. This is feasible with-FIB fabricated-sub-100-nm slanted slits that allow tunnelling.

The optimum grating for $10^{\circ}$ coupling has 87.5 -nm-wide slits at an angle of 58.4 to the surface normal. The fiber is mounted at $10^{\circ}$ to ensure a low second-order reflection in the waveguide. Our simulations were performed on a $25 \mu \mathrm{m} \times 14 \mu \mathrm{m}$ base, and converged with a $10-\mathrm{nm}$ mesh. A plot of the calculated field pattern at a wavelength of $1550 \mathrm{~nm}$ is shown in Fig. 2. The slanted grating coupler has a fiber-to-chip coupling efficiency of $64 \%$, a period of $675 \mathrm{~nm}$, and a $3-\mathrm{dB}$ bandwidth of $100 \mathrm{~nm}$. Fig. 3 shows the calculated power fractions that are diffracted upwards, coupled into the fiber, and reflected in the waveguide. For 1550-nm light the back reflection is only $7 \%$. The total amount of power diffracted out of plane is $83 \%$ at $1550 \mathrm{~nm}$. Although only part

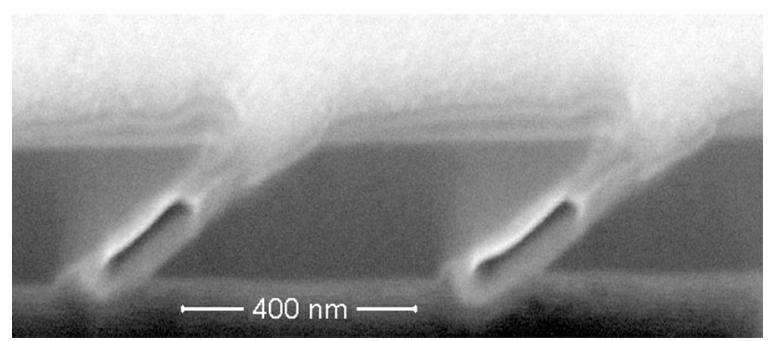

Fig. 4. Cross section of two slits of the slanted grating coupler. There is a good agreement with the FDTD designed grating.

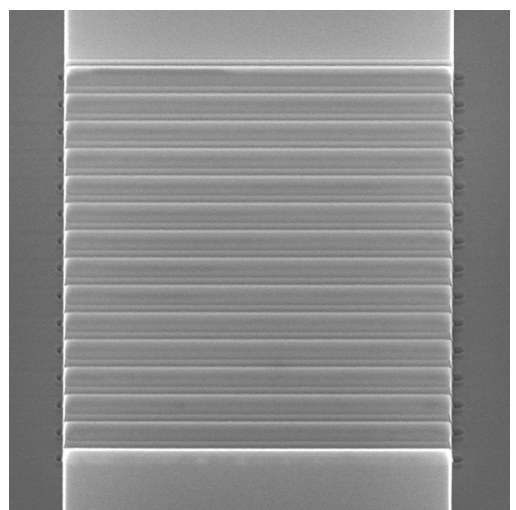

Fig. 5. Top view of the slanted grating coupler.

of this upward power is coupled into the fiber due to mode mismatch for a periodic grating, the mode mismatch can be decreased by varying slit width. However, due to fabrication complexity of varying slit widths, we have chosen only to design and fabricate periodic slanted gratings.

\section{FABRICATION WITH FIB}

In previous work, we have demonstrated that low-loss fiber couplers can be fabricated with FIB [9]. By using $\mathrm{Al}_{2} \mathrm{O}_{3}$ as hard mask and $\mathrm{I}_{2}$ as selective etchant, the loss by crystal damage can be minimized. $\mathrm{Al}_{2} \mathrm{O}_{3}$ has a very low penetration depth for incident gallium ions and has a very low etch rate under iodine atmosphere.

Therefore, $50 \mathrm{~nm}$ of $\mathrm{Al}_{2} \mathrm{O}_{3}$ was deposited on predefined SOI waveguides using electron beam evaporation. Simulations and experiments have shown that this thin layer has no influence on the propagation losses of light in the predefined waveguides. That is why we did not incorporate the layer in our simulations. To etch narrow slanted slits, we have mounted the sample under $58^{\circ}$ relative to the ion beam, and scanned lines under an iodine atmosphere. Both hard mask and silicon are etched in the same run, where narrow slits are formed due to the large etch rate difference between alumina and silicon. The etch dose was optimized to etch down to the oxide buffer layer. We have noticed that the slit width depends strongly on the beam size. The beam current used was $50 \mathrm{pA}$, corresponding to a beam size of about $30 \mathrm{~nm}$. A cross section of two grating slits is shown in Fig. 4, and a top view is shown in Fig. 5. For an etch dose of $1.1 \times 10^{13} \mathrm{Ga}^{+} / \mathrm{cm}$, the slits are etched down to the oxide buffer layer and the slit width corresponds to the simulations. The etch time for 15 slits of $10 \mu \mathrm{m}$ wide is about $8 \mathrm{~min}$. After 


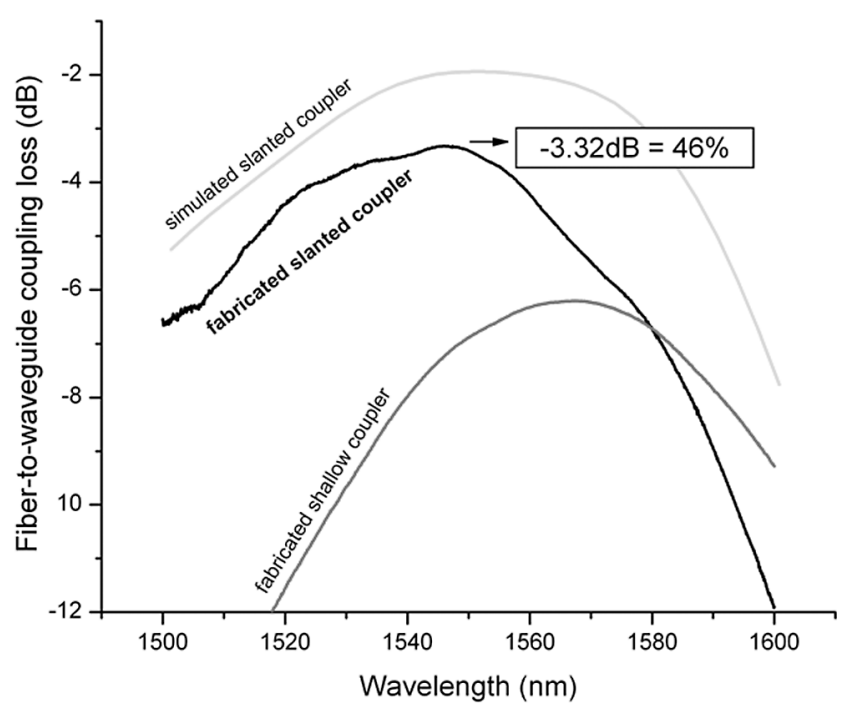

Fig. 6. Coupling spectra of simulated and fabricated slanted fiber coupler, as compared to a shallow grating coupler fabricated with optical lithography.

fabrication, the sample was baked for $2 \mathrm{~h}$ at $300{ }^{\circ} \mathrm{C}$ in a nitrogen atmosphere.

\section{MEASUREMENT AND DISCUSSION}

To determine the coupling efficiency of the fabricated coupling structure, we have used a fiber-to-fiber transmission measurement for TE polarized light from a superluminescent light-emitting diode. The structure consisted of a regular shallow input coupler fabricated with optical lithography, a broad $10-\mu \mathrm{m}$ waveguide, and a slanted output coupler fabricated in situ with FIB. The coupling characteristic of the shallow couplers was measured in a separate setup with identical input and output couplers. The coupling efficiency of the slanted grating coupler is depicted in Fig. 6. It is calculated by subtracting-on a logarithmic scale-source spectrum and shallow coupler spectrum from the measured spectrum. We have extracted a maximum fiber-to-waveguide coupling efficiency of $46 \%$ and a $3-\mathrm{dB}$ bandwidth of about $80 \mathrm{~nm}$ for the slanted fiber coupler fabricated with FIB.

To evaluate the discrepancy between simulated and measured coupling efficiency, we have investigated the fabrication tolerances of the slanted coupler. Period, slant angle, and slot width were varied within the measurement tolerance of the cross section in Fig. 4. The observed drop in efficiency was below 5\%, therefore, bad grating parameters cannot fully explain the discrepancy. Instead, we consider the measured loss to be material related. Is is known that FIB etching generates optical losses in silicon [9]. Although the use of alumina as mask and iodine as enhancement gas considerably reduces these losses, we have noticed that an additional baking step at $300{ }^{\circ} \mathrm{C}$ is necessary to fabricate slanted couplers with efficiencies above $20 \%$. We think that the baking step removes all remaining iodine and silicon iodide from the etched region. However, the damage caused to the silicon crystal remains unaltered after a temperature treatment at $300{ }^{\circ} \mathrm{C}$. Therefore, we are convinced that silicon crystal damage in the region of the etched slits causes the discrepancy between theory and experiment.

\section{CONCLUSION}

We have reported on the design and FIB fabrication of a slanted grating coupler in an SOI waveguide. The coupler has a maximum measured coupling efficiency of $46 \%$ and can be etched in situ, anywhere on a wafer, in less than $10 \mathrm{~min}$.

\section{REFERENCES}

[1] W. Bogaerts, R. Baets, P. Dumon, V. Wiaux, S. Beckx, D. Taillaert, B. Luyssaert, J. Van Campenhout, P. Bienstman, and D. Van Thourhout, "Nanophotonic waveguides in silicon-on-insulator fabricated with CMOS technology," J. Lightw. Technol., vol. 23, no. 1, pp. 401-412, Jan. 2005.

[2] D. Taillaert, P. Bienstman, and R. Baets, "Compact efficient broadband grating coupler for silicon-on-insulator waveguides," Opt. Lett., vol. 29, no. 23, pp. 2749-2751, 2004.

[3] F. V. Laere, G. Roelkens, J. Schrauwen, D. Taillaert, P. Dumon, W. Bogaerts, D. V. Thourhout, and R. Baets, "Compact grating couplers between optical fibers and silicon-on-insulator photonic wire waveguides with 69\% coupling efficiency," in OFC 2006, U.S., 2006, pp. PDP15-PDP15.

[4] G. Roelkens, D. V. Thourhout, and R. Baets, "High efficiency silicon-on-insulator grating coupler based on a polysilicon overlay," Opt. Express, vol. 14, no. 24, pp. 11622-11630, 2006.

[5] S. Rubanov and P. R. Munroe, "Fib-induced damage in silicon," J. Microscopy-Oxford, vol. 214, pp. 213-221, 2004.

[6] A. A. Tseng, "Recent developments in micromilling using focused ion beam technology," J. Micromech. Microeng., vol. 14, no. 4, pp. R15-R34, 2004.

[7] K. E. Zinoviev and C. Dominguez, "Diffraction grating couplers milled in $\mathrm{Si}_{3} \mathrm{~N}_{4}$ rib waveguides with a focused ion beam," Opt. Express, vol. 13 , pp. 8618-8618, 2005.

[8] Y. Tanaka, M. Tymczenko, T. Asano, and S. Noda, "Fabrication of two-dimensional photonic crystal slab point-defect cavity employing local three-dimensional structures," Jpn. J. Appl. Phys., vol. 45, pp. 6096-6102, 2006.

[9] J. Schrauwen, D. V. Thourhout, and R. Baets, "Focused-ion-beam fabricated vertical fiber couplers in silicon-on-insulator waveguides," Appl. Phys. Lett., vol. 89, pp. 141102-141102, 2006.

[10] B. Wang, J. H. Jiang, and G. P. Nordin, "Embedded, slanted grating for vertical coupling between fibers and silicon-on-insulator planar waveguides," IEEE Photon. Technol. Lett., vol. 17, no. 9, pp. 1884-1886, Sep. 2005. 